\title{
CPD questionnaire
}

\section{October 2020}

Nursing students' perceptions and experiences of concept mapping as a learning tool in a human physiology course
1. Participants in this study felt that concept mapping:
A. Promoted group work.
B. Facilitated a deep learning approach.
C. Improved their assimilation of knowledge.
D. A and B.
E. All of the above.

Demystifying sexual connotations: A model for facilitating the teaching of intimate care to nursing students in South Africa (SA)

2. The main concept in this study was defined using the following steps:

A. The dictionary definition, subject definition and exemplary case.

B. The subject definition, dictionary definition and exemplary case.

C. The dictionary definition, exemplary case and subject definition.

D. The exemplary case, dictionary definition and subject definition.

The contribution of nursing preceptors to the future nursing workforce

3. The first three steps of Mey and Dietrich comprise:

A. Contextualisation, description and coding.

B. Contextualisation, description and segmentation.

C. Description, segmentation and coding.

D. Description, coding and interpretation.

Improving postgraduate nursing research output: An SA nursing science perspective

4. Difficulties in exposing student nurses to research include:

A. Excessive workload.

B. Poor time management.

C. Poor programme management.

D. A and B.

E. All of the above.

A broken triangle: Students' perceptions regarding the learning of nursing administration in a low-resource setting

5. The main themes emerging from this study are:

A. Cognitive support during learning.

B. Alignment of assessment with reality.

C. Validity of assessment tools.

D. Achieving learning outcomes.

E. All of the above.
The influence of context on the teaching and learning of undergraduate nursing students: A scoping review

6. In this study, organisational climate and organisational culture were regarded as interchangeable.

A review of geriatric care training in the undergraduate nursing and medical curricula at the University of KwaZulu-Natal, SA

7. Nursing students in this study had clinical exposure to the following settings:

A. Community.

B. Primary care.

C. Residential facilities.

D. Hospitals.

E. A, B, C and D.

F. All of the above.

Effect of a teaching programme on knowledge of postoperative pain management among nurses at Lagos University Teaching Hospital, Nigeria

8. The results of this study showed that there was:

A. No improvement in knowledge in the experimental group after the intervention.

B. A relationship between the teaching programme and the knowledge of postoperative pain management.

C. A relationship between the teaching programme and the knowledge of pain assessment.

D. All of the above.

Factors contributing to poor performance of student nurses in anatomy and physiology

9. The findings of this study revealed that student failure in anatomy and physiology is related to:

A. Poor teaching strategies.

B. Short study periods.

C. Language barriers.

D. Workload.

Predicting effect of emotional-social intelligence (ESI) on academic achievement of nursing students

10. Some of the reasons provided for the satisfactory ESI levels of more than two-thirds of the participants were:

A. Extracurricular activities.

B. Cultural influences.

C. Increased attention to emotional domains in teaching.

D. Summer courses.

A maximum of 3 CEUs will be awarded per correctly completed test.

The CPD programme for AJHPE is administered by Medical Practice Consulting.

CPD questionnaires must be completed online at www.mpconsulting.co.za

After submission you can check the answers and print your certificate.

Questions may be answered up to 6 months after publication of each issue.

Accreditation number: MDB015/006/01/2020 (Clinical) 


\section{CPD Questionnaire}

\section{October 2020}

Liberalisation of education in Cameroon: The liberating-paralysing impact on nursing education

11. In this study, the theme of advancement included the following subcategories:

A. Increased access.

B. Policy controversies.

C. Status recognition.

D. Personal prejudices.
Competencies for structured professional development of neonatal nurses in SA

12. The competency framework for the professional development of different categories of nurses in neonatal practice was based on Benner's five stages of development. 